\title{
Osteosarcoma: A Case Report
}

\author{
Esra Yeșilovaa, \\ Faruk Akgünlüb, \\ Doḡan Dolanmazc, \\ Füsun Yașard, \\ Sevgi Sener
}

\begin{abstract}
Although osteosarcoma of the head and neck is a relatively rare disease, it is a highly malignant bone tumor. Diagnosis of the tumor is important especially in early stages for improving prognosis. The patients with orofacial pain firstly prefer to go to dentists. Such kind of pain may be associated with an oral or maxillofacial cancer. The dentists must be careful for evaluating the clinical and radiologic clues. These clues determine the biopsy necessity, type and management plan. The aim of this case presentation is to emphasize the importance of dentists on diagnosis and prognosis of oral malignancies. (Eur J Dent 2007;1:60-63)
\end{abstract}

Key words: Panoramic radiography; Osteogenic sarcoma; Diagnosis.

\section{INTRODUCTION}

The term osteosarcoma refers to a heterogenous group of primary malignant neoplasms affecting bone forming or mesenchymal tissues that have histopathologic evidence of osteogenic differentiation. ${ }^{1}$ Osteosarcoma is a highly malignant bone tumor ${ }^{2}$ and it is relatively a rare disease in the head and neck region. ${ }^{3}$ It occurs infrequently in the jaws, as some series have revealed that ap-

$\square$ a PhD, Selcuk University, Faculty of Dentistry, Department of Oral Diagnosis \& Radiology; Konya/TURKEY.

b Prof. Dr, Selcuk University, Faculty of Dentistry, Department of Oral Diagnosis \& Radiology; Konya/TURKEY.

'Assoc. Prof. Dr, Selcuk University, Faculty of Dentistry, Department of Oral \& Maxillofacial Surgery; Konya/TURKEY.

${ }^{d}$ Assistant Prof. Dr., Selcuk University,

Faculty of Dentistry, Department of Oral Diagnosis \& Radiology; Konya/TURKEY.

e Assistant Prof. Dr., Selcuk University, Faculty of Dentistry, Department of Oral Diagnosis \& Radiology; Konya/TURKEY.

$\square$ Corresponding author: Dr Esra YEȘiLOVA Selcuk University, Faculty of Dentistry Oral Diagnosis \& Radiology Department KONYA/TURKEY

Tel: +90 3322231255

Fax: +903322410062

e-mail:dtesralahotmail.com proximately only $8 \%$ of all osteosarcomas develop in this location. ${ }^{2}$ Osteosarcomas and chondrosarcomas of the jaws exhibit some behavioral features that separate them from the lesions of the rest of the skeleton. Jaw lesions tend to occur at an older mean age (fourth decade versus second decade for non jaw lesions). ${ }^{4}$

Pain and swelling are more typical of jaw lesions. ${ }^{4}$ Metastasis is less likely ${ }^{2,4}$ and prognosis is correspondingly more favorable: approximately $40 \% 5$-year survival rate versus $20 \%$ for non jaw lesions. $^{4}$

Although in the jaws conventional osteosarcomas involving the mandible and maxilla display a predilection for males, ${ }^{2,4,4,6,-8}$ some studies display slight predilection for females. ${ }^{9,10}$ There is a nearly equal involvement of the maxilla and the mandible. $2.5,6$ Bennett et al ${ }^{9}$ stated that mandibular tumors were predominant in females. In contrast, Forteza et $\mathrm{a}^{10}$ stated that the maxillary tumors were predominant in females. In addition, most maxillary lesions were found osteoblastic and mandibular lesions were found osteolytic. The majority of mandibular osteosarcomas arise in the body of the mandible; the remaining sites of the predilection include the symphisis, angle of the mandible, ascending ramus and temporomandibular joint. In the maxilla, there is a nearly equal in- 
cidence of tumors involving the alveolar ridge and maxillary antrum, with a few affecting the palate. ${ }^{5}$ Maxillary tumors may extend to the infratemporal fossa and to maxillary sinus and attain a greater volume before diagnosis. ${ }^{6}$ In differential diagnosis of osteosarcoma, fibrous dysplasia and osteomyelitis must be taken into consideration. ${ }^{11}$

In this article, we want to emphasize the importance of dentists on diagnosis and prognosis of oral malignancies with presenting an osteosarcoma case.

\section{CASE REPORT}

A fifty year-old woman referred to Selcuk University, Faculty of Dentistry, Oral Diagnosis and Radiology Department Clinic with a chief complaint of a remaining root and pain which had been lasting for two months after a tooth extraction from right maxillary posterior region. She stated that she had her teeth extracted because of a long lasting pain, but until that time her pain had not relieved. She had no medical problem.

Intra oral examination revealed a $2 \mathrm{~cm}$ sized, fibrotic, non bleeding mass coinciding with a dense expansion in bone (Figure 1). The lesion on the alveolar ridge was in lobular form. Although muco-

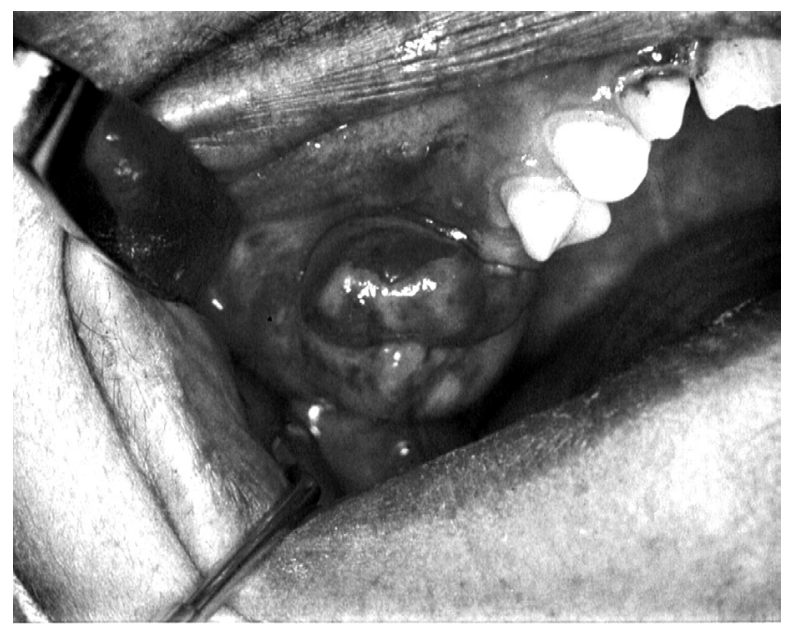

Figure 1. The soft tissue mass is seen on the right side of maxillary alveolar crest on intraoral examination.

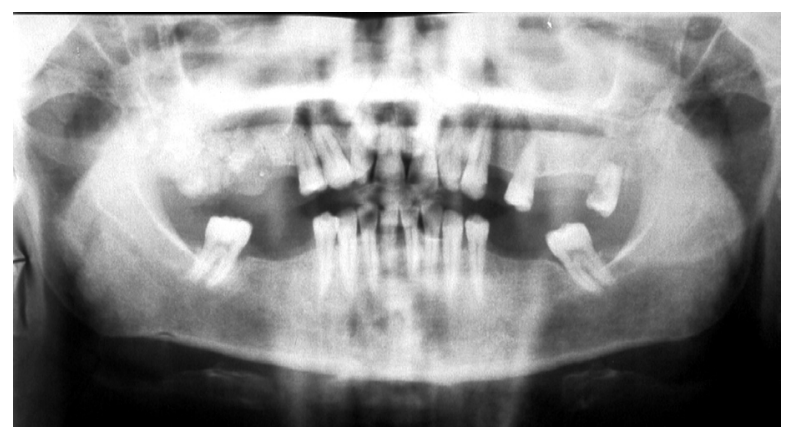

Figure 2. Panoramic radiography revealed "sun burst" appearance of tumour on right side of the mandible. The resorption of the apex of left maxillary second molar tooth is also seen in radiograph. sitis areas were found owing to occlusal trauma, there was no ulceration on the surface of the lesion. She said that the lesion had become larger in two months. There was no lymphadenopaty. She had no sensory or motor deficiency.

On the panoramic radiography (Figure 2), the lesion was seen as extending to tuber maxilla distally and to maxillary sinus coronally. The inferior border of the maxillary sinus couldn't clearly be followed on distal side. The radiographic dimensions of the lesion were approximately $4 \times 3 \mathrm{~cm}$ and the borders were ill-defined. The "sun-burst appearance", which is one of the characteristic properties of malign lesions, could easily be seen. On the left side, the apex of left maxillary second molar tooth was seen as resorpted. A lytic area was seen between apex and the surrounding bone. The lesion was thought as malignant because of the radiological findings, clinical appearance and the history of the patient. The tentative diagnoses were chondrosarcoma and osteosarcoma.

Firstly, an incisional biopsy was taken under local anesthesia. The pathologic examination revealed calcifying fibroma. But in the view of clinical and radiological features of the case, calcifying fibroma result seemed to be unharmonious. Therefore it was decided to take a second biopsy. Another surgeon took a deep biopsy specimen particularly including bone tissue. The second histopathologic examination revealed osteoblastic osteosarcoma (Figure 3). Patient was sent to an otorhinolaryngology department for oncologic surgery and management.

\section{DISCUSSION}

Our case is in agreement with the literature on age, pain, swelling, ${ }^{4}$ gender,, 90 tumor type ${ }^{10}$ and location, 5,6 presenting signs of the disease. ${ }^{4}$ Also the tumor extended to maxillary sinus before being diagnosed.

Histopathologic appearances of osteosarcoma, osteomyelitis and fibrous dysplasia occupy a spec-

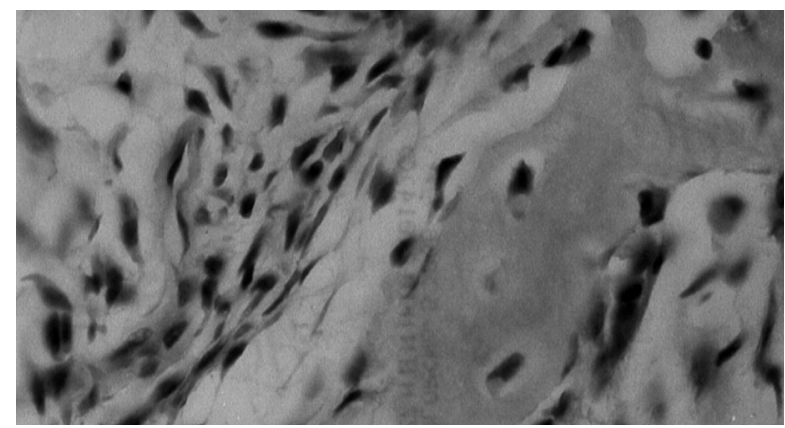

Figure 3. Microscopic examination of tumoral tissue (HE and $\times 40)$. 
trum that may have considerable overlap. In some cases, a classic histopathologic appearance makes the diagnosis clear; however, when the picture is that of new bone formation in a back-ground of cellular fibrous connective tissue, the diagnosis is more difficult. Depending on tissue sampling and location of biopsy, these same features could occur in well-differentiated (fibroblastic) osteosarcoma, active fibrous dysplasia and chronic osteomyelitis. In making diagnosis, the clinician must weigh all available diagnostic information. In these lesions, the radiographic characteristics can play a more significant role especially when histopathology is not clear-cut and classic. ${ }^{11}$

The overlapped radiographic characteristics of these lesions are:

- All of them frequently occur in the posterior body of the mandible.

- The periphery may be ill defined and they can all cause enlargement.

- Internal structure is commonly a mixture of radiolucency and radiopacity. The new bone formed may present a granular stippled pattern.

The distinguishing features are:

- Osteosarcoma may destroy cortical boundaries. It causes widening of periodontal ligament space. This may result in a spiculated pattern of new bone formation in association with a soft tissue mass peripheral to outer cortical boundary of the involved bone. ${ }^{11}$ This finding was also seen in our case.

- Fibrous dysplasia can cause expansion of the involved bone but maintain a thinned, intact cortical boundary. Fibrous dysplasia can alter the appearance of lamina dura but the periodontal ligament space is not widened. ${ }^{11}$ Fibrous dysplasia has different radiographic patterns such as ground-glass, orange peel and cotton wool. ${ }^{12}$ Also because fibrous dysplasia is usually found in more younger age group than osteosarcoma, in our tentative diagnosis it was excluded.

- Osteomyelitis shows the presence of sequestra and laminated periosteal reactive bone formation usually. ${ }^{11}$ No infection finding was found in our case.

Computed tomography has come to play a large role in the interpretation of osseous changes in the jaws. ${ }^{13}$ But the diagnosis of dentists are especially confined to plain films. The panoramic radiography is commonly used in dental practice as well as periapical and occlusal radiography and is well interpreted by general practitioners and dental specialists. These practitioners are often the first to examine patients with osteogenic sarcoma. Thorough radiological examination and recognition of the radiological features of osteogenic sarcoma can lead to a prompt diagnosis. This will lead to earlier referral of patients for definitive diagnosis and may prevent needless tooth extraction, which facilitates the spread of the tumour. ${ }^{8}$ The teeth of patients with osteosarcoma may displace or be lost. $^{2}$ But there are different causes of tooth loss such as periodontal and endodontic ones. ${ }^{14}$ Radiographic evaluation is important in diagnosis, because clinical symptoms, such as pain, paresthesia, swelling and loosening of teeth are not specific. ${ }^{10}$ Better knowledge and understanding of the radiological features of can lead to an earlier diagnosis, shorten any delay and so improve the prognosis. ${ }^{8}$ The teeth of our patient were extracted because of insufficient evaluation the condition. Therefore early diagnosis may improve the patients' life and obtain to live them with minimal defects. However, as in our case, the operation of the tumoral tissue will be difficult due to the proximity of important anatomical structures. In addition the insidious progress of the lesion invites us to be careful in early diagnosis.

Consequently, knowing the radiological and clinical properties of malignant lesions will provide the determination of biopsy necessity and type. In the first biopsy, superficial specimen was taken. By comparison of histopathological results, it can be said that in the lesions which extended from central to peripheral tissues and affected the soft tissues, biopsy specimen should be taken not only from soft tissue but also from bone. Petrikowski et $\mathrm{al}^{11}$ compared the osteosarcoma, fibrous dysplasia and osteomyelitis. In the majority of their osteosarcoma cases they saw spiculations. They concluded that a periosteal response consisting of spiculations perpendicular to the periosteum is often associated with malignant bone disease although it is not a consistent finding particularly early in lesion maturation when periosteal involvement has not yet occurred. ${ }^{11}$ In our case the superficial specimen that included perhaps immatured part of the lesion caused the pathologist to decide to define the lesion as calcifying fibroma.

Oral cancer is best managed through a board or team. Such a team might consist of a dental hygienist, dentist, dental specialist, oncologists, nutritionist, psychiatrist or psychologist and social worker. The dental community has a responsibility for education, early detection, diagnosis and referral of cancer to confreres competent in the treatment of oral cancer. ${ }^{15}$

\section{CONCLUSIONS}

The patients firstly prefer to go to the dentists when they have an orofacial pain. Such kind of pain may be associated with an oral or maxillofacial cancer. So the dentists are important in the diagnosis of not only oral but also maxillofacial 
lesions and they should be able to distinguish lesions especially malign ones by combining clinical and radiological findings.

\section{REFERENCES}

1. Schajowicz F, Sissons HA, Sobin LH. The World Health Organization's histologic classification of bone tumors. A commentary on the second edition. Cancer 1995;75:12081214.

2. Clark JL, Unni KK, Dahlin DC, Devine KD. Osteosarcoma of the jaw. Cancer 1983;51:2311-2316.

3. Oda D, Bavisotto LM, Schmidt RA, McNutt M, Bruckner JD, Conrad EU 3rd, Weymuller EA Jr. Head and neck osteosarcoma at the University of Washington. Head Neck 1997; 19:513-523.

4. Regezi JA, Zarbo RJ, McClatchey KD, Courtney RM, Crissman JD. Osteosarcomas and chondrosarcoma of the jaws: Immunohistochemical correlations. Oral Surg Oral Med Oral Pathol 1987:64:302-307.

5. Zarbo RJ. Malignant Non-odontogenic Neoplasms of the Jaws. In: Regezi JA, Schuibba J (eds.) Oral Pathology Clinical Pathologic Correlations. 2nd ed. pp 436-457. Philadelphia, Pennsylvania: W.B. Saunders Company, 1993.

6. Mardinger O, Givol N, Talmi YP, Taicher S. Osteosarcoma of the jaw, The Chaim Sheba Medical Center experience. Oral Surg Oral Med Oral Pathol Oral Radiol Endod 2001;91. 445-451.

7. Bianchi SD, Boccardi A. Radiological aspects of osteosarcoma of the jaws. Dentomaxillofac Radiol 1999;28:42-47.

8. Givol N, Buchner A, Taicher S, Kaffe I. Radiological features of osteogenic sarcoma of the jaws. A comparative study of different radiographic modalities. Dentomaxillofac Radiol 1998:27:313-320.

9. Bennett JH, Thomas G, Evans AW, Speight PM. Osteosarcoma of the jaws: A 30-year retrospective review. Oral Surg Oral Med Oral Pathol Oral Radiol Endod 2000;90:323-333.

10. Forteza G, Colmenero B, Lopez-Barea F. Osteogenic sarcoma of the maxilla and mandible. Oral Surg Oral Med Oral Pathol 1986;62:179-184.

11. Petrikowski CG, Pharoah MJ, Lee L, Grace MG. Radiographic differentiation of osteogenic sarcoma, osteomyelitis, and fibrous dysplasia of the jaws. Oral Surg Oral Med Oral Pathol Oral Radiol Endod 1995:80:744-750.

12. White SC, Pharoah MJ. Diseases in bone manifested in the jaws. In: White SC, Pharoah MJ (eds). Oral Radiology Principles and Interpretation. 4th ed. pp 444-471. Missouri: Mosby-Year Book, 2000.

13. Cavalcanti MG, Ruprecht A, Yang J. Radiological findings in an unusual osteosarcoma in the maxilla. Dentomaxillofac Radiol 2000;29:180-184.

14. Richards W, Ameen J, Coll AM, Higgs G. Reasons for tooth extraction in four general dental practices in South Wales. Br Dent J 2005; 198:275-278.

15. Wood NK, Sawyer DR. Oral Cancer. In: Wood NK, Goaz PW (eds.) Differential Diagnosis of Oral and Maxillofacial Lesions. 5th ed. pp 587-595. St. Louis, Missouri: Mosby-Year Book, 1997. 